1Dr. Fahmida Ferdous Assistant Professor Dept. of Psychiatry Z. H. Sikder Womens' Medical College, Dhaka

${ }^{2}$ Prof. Dr. B H Nazma Yasmeen

Professor and Head

Dept. of Pediatrics

Northern International Medical College, Dhaka

3 Prof. Md. Faruq Alam

Professor \& Director

National Institute of Mental Health

${ }^{4}$ Dr. Md. Mahsukur Rahman Chisty Senior Lecturer

Dept. of Anatomy

Dhaka National Medical College

${ }^{5}$ Prof. Md. Mohit Kamal

Professor and Head

Dept. of Psychotherapy

National Institute of Mental Health

${ }^{6}$ Prof. Jinnath Imtiaz Ali

Professor

Dept. of Linguistics

University of Dhaka

\section{Correspondence}

Dr. Fahmida Ferdous

Assistant Professor

Dept. of Psychiatry

Z. H. Sikder Womens' Medical

College

e-mail: ferdous_fahmida@yahoo.com

\title{
Language Problem in Bangladeshi Children with Autism spectrum disorder : Observation through a Neurolinguistics Approach
}

\author{
F Ferdous ${ }^{1}$, B H N Yasmeen ${ }^{2}$, M F Alam ${ }^{3}$, M M R Chisty ${ }^{4}$, M M Kamal $^{5}$, J I Ali ${ }^{6}$
}

\section{Abstract}

Background : Autism spectrum disorder (ASD) covers a set of developmental disabilities that can cause significant social, communication, and behavioral challenges.

Objective : It was aimed to find out the language problem in Bangladeshi children with Autism spectrum disorder following the neurolinguistics approach.

Methodology : A descriptive observational study conducted during the period of April 2014 to December 2014 in the department of Linguistics, University of Dhaka, Bangladesh among the Bangladeshi children aged between 3-12 years. A total of 10 children with normal hearing ability who were diagnosed as ASD according to the diagnostic criteria (DSM5 or ICD 10) were enrolled in the study purposively from two tertiary care hospitals of Dhaka city.

Results : In this study total 10 children were included and their age range was 3 to 12 years. Male was predominant $(90 \%)$.Consanguinity of parents was not present in most $(90 \%)$ cases, $60 \%$ had positive family history of mental illness, $70 \%$ had history of delivery by lower uterine section, $80 \%$ of them were term baby. $100 \%$ children had delayed speech development, $90 \%$ was unable to indicate with index finger, $90 \%$ was unable to use noun and pronoun, $90 \%$ had repetitive words, $100 \%$ had inability to take part in rhymes, $90 \%$ was unable to play with symbols, $70 \%$ had no eye to eye contact, and $90 \%$ had no reciprocal social smile.

Conclusion : Language problem in the children with ASD is an under attended problem in Bangladesh. To the authors' best knowledge this is the first neurolinguistics approach to ASD children in Bangladesh. Further large scale extensive studies would be necessary for better visualization of the extent of the issue.

Key Words : Neurolinguistics, Autism spectrum disorder, Bangladeshi.

DOI: http://dx.doi.org/10.3329/nimcj.v9i2.38910

Northern International Medical College Journal Vol. 9 No. 2 January 2018, Page 295-299

\section{Introduction}

Autism spectrum disorder (ASD) covers a set of developmental disabilities that can cause significant social, communication, and behavioral challenges. ${ }^{1-3}$ People with ASD process information in their brain differently than other people. They share some symptoms, such as difficulties with social interaction, but there are differences when the symptoms start, how severe they are, how many symptoms there are, and whether other problems are present.

The signs of ASD begin before the age of 3 years, although some children may show hints of future problems within the first year of life. ${ }^{1-3}$ Children with ASD often are self-absorbed and seem to exist in a private world where they are unable to successfully communicate and interact with others. They may have difficulty in developing language skills and understanding what others say to them. They also may have difficulty in communicating nonverbally, such as through gestures, eye contact, and facial expressions etc. ${ }^{1-3}$ Most children with ASD have little or no problem pronouncing words. The majority, however, have difficulty ensuing language effectively, especially when they talk to other people. ${ }^{2-3}$ Many have problems with the meaning and rhythm of words and sentences. They also may be unable to understand body language and the nuances of vocal tones. ${ }^{3}$

Individual with ASD put a heavy demand on educational, social and medical services, and accurate prevalence estimates are needed for the planning of such services. Until the 1990s, the figure of 4 to 5 cases of autism per 10000 people was widely accepted, although as many as 20 per 10000 children were reported as showing the triad of impairments in social reciprocity, language impairment and reduced imagination and restricted activities. ${ }^{4}$ Studies 
have shown increased prevalence estimates for all ASDs of between 30 and 90 cases per $10000 .^{5-9}$ In addition to a true increase in prevalence, alternative explanations have been proposed, including changing diagnostic criteria, different methods of ascertainment, varying urban, rural, and country location, and population of study, younger age, and inclusion of individuals with average intelligence quotient (IQ) and those with other neuropsychiatric and medical disorders. ${ }^{3,7}$ Individual with ASD may have problems impeding their development of speech and language that are well outside the scope of traditional speech and language therapy (such as social deficits) or, at the very least, in the very frontiers of clinical knowledge as to appropriate treatment (developmental articulation disorders). ${ }^{1,2}$

Parents and teachers are confronted by a bewildering range of options and apparent philosophies of treatment of these individuals. Thus, identifying and treating specific speech and language problems at the earliest possible age is of the utmost importance in children with autism.

Neurolinguistics is the study of how language is represented in the brain: that is, how and where our brains store our knowledge of the language (or languages) that we speak, understand, read, and write, what happens in our brains as we acquire that knowledge and what happens as we use it in our everyday lives. Neurolinguistics try to answer questions like these: What about our brains makes human language possible. ${ }^{10}$

To date, research on speech and language development in children with ASD has been limited, and dearth of literature in the developing countries like Bangladesh. The language problem in ASD children is still under attended though we have huge population with a significant portion of growing children in Bangladesh.

Therefore, we aimed to find out the language problem in children with ASD in Bangladesh following the basis of neurolinguistics approach.

\section{Methodology}

This was a neurolinguistics study conducted during the period of April 2014 to December 2014 at the department of Linguistics, University of Dhaka among the Bangladeshi children aged between 3-12 years. A total of 10 consecutive children with ASD were enrolled in the study on the basis of the inclusion criteria.

\section{inclusion criteria}

- Children who have hearing abilities within normal limits as documented by audio logical evaluation by Audiologist.

- Children who diagnosed as ASD according to the diagnostic criteria (DSM-5 or ICD 10) for the first time at the tertiary center and had no previous interventions.

\section{Exclusion criteria}

- Children who have got previous interventions were excluded from the study.
Sampling was done purposively from two tertiary care hospitals of Dhaka city (National Institute of Mental Health, Sir Salimullah Medical College). Informed consent was taken from all the parents or attendant before enrollment in the study.

Data were collected using predeveloped questionnaire. The questionnaire was composed of 18 questions, which was developed for find out the linguistics output in children with ASD.

In this study a questionnaire of 18 questions were used to find out the linguistics output of children with ASD. All these questions covered the Receptive language development, Expressive language development and Social communication of child with ASD. In the questionnaire 3 questions were covered the receptive language development, 8 questions covered the expressive language development and further 7 questions covered the social communication.

Data were collected on clinical observation and history taking from mother/care giver, following linguistics approach Data analysis was done by using SPSS, Version-16

\section{Results}

In this study regarding the distribution of demographic variables of the children with ASD it was observed that majority $(60 \%)$ of the patients were found in 3-6 years age group and rest 4 $(40 \%)$ as found in $>6-9$ years group. It was observed that male was found 9 (90\%) and female was 1 (10.0\%). The respondents' relation to patients was mother $8(80 \%)$, father was $2(20 \%)$. History of consanguinity was found in $1(10 \%)$ respondents and $9(90 \%)$ of the children had non consanguineous parents. 8 (80\%) had term pregnancy, $1(10 \%)$ had preterm pregnancy and $1(10 \%)$ had post term pregnancy. Three (30\%) had normal vaginal deliver (NVD) and $7(70 \%)$ had lower uterine section (CS) delivery (Table 1).

$\begin{array}{lcc}\begin{array}{l}\text { Table I : Distribution of demographic variables of the ASD patients } \\ \text { Demographic Variable }\end{array} & \text { Frequency } & \text { Percentage } \\ \begin{array}{l}\text { Age in Years } \\ 3-6\end{array} & 6 & 60 \\ >6-9 & 4 & 40 \\ >9-12 & 0 & 0 \\ \text { Sex } & & \\ \text { Male } & 9 & 90 \\ \text { Female } & 1 & 10 \\ \text { Respondents relation to patient } & & \\ \text { Mother } & 8 & 80 \\ \text { Father } & 2 & 20 \\ \text { History of consanguinity } & & \\ \text { Yes } & 1 & 10 \\ \text { No } & 9 & 90 \\ \text { Family history of mental illness } & & \\ \text { Yes } & 6 & 60 \\ \text { No } & 4 & 40 \\ \text { Gestational age } & & \\ \text { Full term } & 8 & 10 \\ \text { Pre-term } & 1 & 10 \\ \text { Post-term } & 1 & 30 \\ \text { Mode of delivery } & & 70 \\ \text { Normal vaginal delivery } & & \\ \text { Lower uterine section } & 3 & \\ & 7 & \end{array}$


On clinical observation and history taking from mother/care giver, regarding Receptive language development following linguistics approach we found that all (100\%) parents thought that their children were not deaf. Hearing was normal in all cases even though most of the children $7(70 \%)$ dose not respond to his/her name. Also most of the study patients 8 $(80 \%)$ could not identify $4 / 5$ body parts (Table II).

\section{Expressive language development}

Regarding assessment of Expressive language development, we used 8 questions and the linguistics output of the study showed that all children (100\%) had speech delay and $6(60 \%)$ children used his/her index finger to point, to indicate interest in sometimes. We found that $9(90 \%)$ children did not ever use his/her index finger to point, when we asked something and could not able to use noun and pronoun and repeatedly utter any words but they did not have any fluency disorder. In our study majority children $8(80 \%)$ could not say name of known object and $7(70 \%)$ children used idiosyncratic/neologism words (Table III).

Table II : Receptive language development

\begin{tabular}{|c|c|c|c|}
\hline Questions & Normal Child & Child with ASD (study patients) & No/ \% \\
\hline $\begin{array}{l}\text { Have you ever thought that your child is deaf? } \\
\text { (আপনার কি কখনো মনে হয় আপনার শিঙটি কানে শনেে না?) }\end{array}$ & Hearing is normal & Hearing is normal & $10(100 \%)$ \\
\hline $\begin{array}{l}\text { 2. Does your child respond to his/her name? } \\
\text { (আপনার শিফ কি তার নাম ধরে ডাকলে সাড়া দেয়?) }\end{array}$ & $\begin{array}{l}\text { Within 9-11 months a normal child } \\
\text { can response to his/her name. }\end{array}$ & dose not respond to his/her name & $7(70 \%)$ \\
\hline $\begin{array}{l}\text { 3. Does your child can identify } 4 / 5 \text { body parts? } \\
\text { e.g point your eyes, nose } \\
\text { (আপনার শিশ্ঞ কি শরীরের } 8 / ৫ \text { টি অঙ্গ নির্দেশ করতে পারে?) } \\
\text { যেমন: নাক, কোথায়, চোখ কোথায় ইত্যাদি }\end{array}$ & $\begin{array}{l}\text { Within } 18-20 \text { months a normal } \\
\text { child can identify } 4 / 5 \text { body parts. }\end{array}$ & could not identify $4 / 5$ body parts & $8(80 \%)$ \\
\hline
\end{tabular}

Table III : Expressive language development

\begin{tabular}{|c|c|c|c|}
\hline Questions & Normal Child & Child with ASD (study patients) & No/ \% \\
\hline $\begin{array}{l}\text { 1. Have you ever thought that your child has speech } \\
\text { developmental delay? } \\
\text { (আপনার কখনো মনে হয়েছে যে আপনার শিশটি কথা বলতে দেরী হয়েছে?) }\end{array}$ & $\begin{array}{l}\text { Within } 4-5 \text { years a normal child has } \\
\text { completed basic language } \\
\text { development. } \\
\text { Within } 12-14 \text { months a normal child } \\
\text { can express meaningful single word. }\end{array}$ & All children had speech delay. & $10(100 \%)$ \\
\hline $\begin{array}{l}\text { 2. Does your child use his/her index finger to point or to } \\
\text { indicate something of his interest? } \\
\text { (আপনার শিঙটি কি কাঙ্গিত বস্তুর দিকে তর্জনী দিয়ে নির্দেশ করতে পারে?) }\end{array}$ & $\begin{array}{l}\text { Within } 12-14 \text { months a normal child } \\
\text { can indicate his/her index finger to } \\
\text { point to indicate interest in } \\
\text { something. }\end{array}$ & $\begin{array}{l}\text { Majority children use his/her } \\
\text { index finger to point, to } \\
\text { indicate something of his } \\
\text { interest }\end{array}$ & $6(60 \%)$ \\
\hline $\begin{array}{l}\text { 3. Does your child ever use his/her index finger to point, } \\
\text { when you ask something? Where is the toy? } \\
\text { (আপনার শিঙটি কি কোন পরিচিত বস্তু সম্পর্কে প্রশ্ন করলে তা তর্জনী নির্দেশ } \\
\text { করে দেখাতে পারে?) উদাহরণ: খেলনাটা কোথায়? }\end{array}$ & $\begin{array}{l}\text { Within } 25 \text { months to } 2.5 \text { years a } \\
\text { normal child can indicate his/her } \\
\text { index finger to point when someone } \\
\text { ask }\end{array}$ & $\begin{array}{l}\text { Most children did not ever use } \\
\text { his/her index finger to point, } \\
\text { when we ask something }\end{array}$ & $9(90 \%)$ \\
\hline $\begin{array}{l}\text { 4. Does your child have Any fluency disorder in speech? } \\
\text { (আপনার শিঙ্টি কি কথা বলার সময় সাবলীলতায় কোন অসুবিধা আছে? } \\
\text { উদাহরণঃ আমি বেড়াতে যাব }\end{array}$ & $\begin{array}{l}\text { child do not have any fluency } \\
\text { disorder }\end{array}$ & $\begin{array}{l}\text { Most children do not have any } \\
\text { fluency disorder }\end{array}$ & $9(90 \%)$ \\
\hline $\begin{array}{l}\text { 5. Does your child can say name of known object? } \\
\text { e.g. Mother, Cat, Dog, Bird etc. } \\
\text { (আপনার শিঞটি কি পরিচিত বস্তুর নাম বলতে পারে?) } \\
\text { উদাহরণ: গাছ, কুকুর, বিড়াল, পাখি, মা }\end{array}$ & $\begin{array}{l}\text { Within } 19-24 \text { months a normal child } \\
\text { can say name of known object. }\end{array}$ & $\begin{array}{l}\text { Most children could not say } \\
\text { name of known object }\end{array}$ & $8(80 \%)$ \\
\hline $\begin{array}{l}\text { 6. Does your child can use 'noun' and 'pronoun' example } \\
\text { "I" "You" "Mine"? How he/she use it? } \\
\text { (আপনার শিঞটি কি “ আমার” “ আমি” শব্দগুলি ব্যবহার করতে পারে? } \\
\text { কিভাবে ব্যবহার করে?) }\end{array}$ & $\begin{array}{l}\text { Within } 25 \text { months to } 2.5 \text { years a } \\
\text { normal child can used noun and } \\
\text { pronoun. } \\
\text { i.e. Q. How are you? } \\
\text { answered : I am fine }\end{array}$ & $\begin{array}{l}\text { Most children could not able } \\
\text { to use noun and pronoun } \\
\text { i.e. Q. How are you? } \\
\text { answered : Anik is fine }\end{array}$ & $9(90 \%)$ \\
\hline $\begin{array}{l}\text { 7. Does your child repeatedly utter any words? } \\
\text { (আপনার শিঙটি কি কোন শব্দ বারবার পুনরাবৃত্তি করে?) }\end{array}$ & $\begin{array}{l}\text { Usually child does not repeatedly } \\
\text { utter any words }\end{array}$ & $\begin{array}{l}\text { Most children repeatedly utter } \\
\text { any words. }\end{array}$ & $9(90 \%)$ \\
\hline $\begin{array}{l}\text { 8. Does your child use idiosyncratic/Neologism words? } \\
\text { (আপনার শিঙ্গটি কি কোন অস্বাভাবিক শব্দ উচ্চারিত করে যার কোন অর্থ নেই?) }\end{array}$ & $\begin{array}{l}\text { Usually child does not use } \\
\text { idiosyncratic/Neologism words }\end{array}$ & $\begin{array}{l}\text { Majority children used } \\
\text { idiosyncratic/neologism words }\end{array}$ & $7(70 \%)$ \\
\hline
\end{tabular}


Regarding assessment of Social communication, we had used 7 questions and the linguistics output of the study showed that all children (100\%) were unable to participate in rhymes/song with other children and could not point or look at things with others jointly. Majority of them (60\%) did not try to attract your/others children with autistic disorder was 2.2 males for each female. ${ }^{6}$ Another review revealed that the most widely reported malefemale ratio for autism prevalence is 4-5:1, lower in individuals with intellectual disability and higher at the high-functioning end. ${ }^{13}$

Table IV : Social communication

\begin{tabular}{|c|c|c|c|}
\hline Questions & Normal Child & (study patients) & No/ $\%$ \\
\hline $\begin{array}{l}\text { 1.Does your child try to attract your/others attention to } \\
\text { his/her activity? (আপনার শিশুটি কি তার কার্যকলাপের দিকে } \\
\text { আপনার/অন্য কারো মনোযোগ আকর্ষণ করতে চেষ্টা করে? }\end{array}$ & $\begin{array}{l}\text { Within } 9-11 \text { months a normal child } \\
\text { can try to attract others attention to } \\
\text { his/her activity. }\end{array}$ & $\begin{array}{l}\text { Majority children did not try to attract } \\
\text { your/others attention to his/her activity }\end{array}$ & $6(60 \%)$ \\
\hline $\begin{array}{l}\text { 2. Does your child participate in rhymes/song with } \\
\text { other children? (আপনার শিঙটি কি অন্য শিঙ্ডের সাথে ছড়া এবং } \\
\text { গানে অংশ নিতে পারে?) }\end{array}$ & $\begin{array}{l}\text { Within } 25 \text { months to } 2.5 \text { years a } \\
\text { normal child can take participate in a } \\
\text { rhymes/song with other children. }\end{array}$ & $\begin{array}{l}\text { All children were unable to take } \\
\text { participate in rhymes/song with other } \\
\text { children }\end{array}$ & $10(100 \%)$ \\
\hline $\begin{array}{l}\text { 3.Does your child ever playing with symbol? } \\
\text { (আপনার শিঝ্টটি কি প্রতীকী খেলা খেলতে পারে?) }\end{array}$ & $\begin{array}{l}\text { Within } 19-24 \text { months a normal child } \\
\text { can playing with a symbol. }\end{array}$ & $\begin{array}{l}\text { Most of the children did not ever } \\
\text { playing with symbol }\end{array}$ & $9(90 \%)$ \\
\hline $\begin{array}{l}\text { 4. Does your child take part in conversation with others? } \\
\text { you take rice? In answering this question whether the } \\
\text { child answer Yes or No } \\
\text { (আপনার শিশটি কি কারো সাথে সংক্ষিপ্ত কথপোকথন চালাতে পারে?) } \\
\text { (উদাহরণঃ ভাত খেয়েছে? এই থ্রশ্নের উত্তর হ্যা বা না বলে কিনা?) }\end{array}$ & $\begin{array}{l}\text { Within } 3 \text { years to } 3.5 \text { years a normal } \\
\text { child can take part in conversation. }\end{array}$ & $\begin{array}{l}\text { Most of the children did not take part } \\
\text { in conversation. }\end{array}$ & $9(90 \%)$ \\
\hline $\begin{array}{l}\text { 5. Does your child can make eye contact and } \\
\text { understand other facial expression? (আপনার শিঙটি কি চোখে } \\
\text { চোখ রাখতে পারে এবং মুখভশি দেখে বক্তার মনোভাব বুঝতে পারে? }\end{array}$ & $\begin{array}{l}\text { Within } 12 \text { - } 14 \text { months a normal child } \\
\text { can make eye contact and understand } \\
\text { others facial expression. }\end{array}$ & $\begin{array}{l}\text { Majority children could not make eye } \\
\text { contact and did not understand others } \\
\text { facial expression }\end{array}$ & $7(70 \%)$ \\
\hline $\begin{array}{l}\text { 6. Does your child point or look at things with other } \\
\text { jointly? (আপনার শিশটি কি অন্যজনের সর্দে একই সাথে কোন } \\
\text { জিনিস নির্দেশ করতে পারে বা দেখাতে পারে?) }\end{array}$ & $\begin{array}{l}\text { Within } 9-11 \text { months a child can point } \\
\text { or look at things with others jointly. }\end{array}$ & $\begin{array}{l}\text { All children could not point or look at } \\
\text { things with others jointly }\end{array}$ & $10(100 \%)$ \\
\hline $\begin{array}{l}\text { 7. Does your child smile in response to your activity } \\
\text { which he/she likes? (আপনার শিশ্টি কি হাসি বিনিময় করে তার } \\
\text { পছন্দ মতো আপনি কিছু করলে) }\end{array}$ & $\begin{array}{l}\text { Within } 15-18 \text { months a child smiles in } \\
\text { response to your activity which he/she } \\
\text { likes. }\end{array}$ & $\begin{array}{l}\text { Majority children did not smile in } \\
\text { response to any activity which he/she } \\
\text { likes }\end{array}$ & $9(90 \%)$ \\
\hline
\end{tabular}

attention to his/her activity and $70 \%$ could not make eye contact and did not understand others facial expression. We found that $9(90 \%)$ children did not ever play with symbol and did not take part in conversation and did not smile in response to any activity which he/she likes (Table IV).

\section{Discussion}

Bangladesh is a developing country having population of 167 million. According the World health Organization supported survey 2009 there are $0.84 \%$ children are suffering from Autism Spectrum disorders. ${ }^{11}$ Studies recommended that among the developmental disorders, developmental language disorder was the second most common and in ASD it is an important part. ${ }^{12}$ With this huge magnitude of disease burden of ASD children, their language problem is still under attended.

Therefore, we aimed to find out the language problem in children with ASD following the basis of neurolinguistics approach and so far our knowledge this might be the first study carried out in Bangladesh in this regards.

In this study total 10 children were included according to inclusion criteria and their age range were found 3 to 6.5 years. Male was predominant, which was $90 \%$. Male to female ratio was 9:1. Bertrand et al. found the male-to-female ratio for
In our study $70 \%$ parents mentioned that their children could not respond to his/her name and $90 \%$ children ever use his/her finger to point, to indicate something of his interest. Seventy percent parents mentioned that their children ever use his/her index finger to point, to ask for something. Sixty percent parents also mentioned that their children did not try to attract others attention to his/her own activity. Only $10 \%$ children could play with symbol, could take part in conversation and have any fluency disorder in speech.

Recent reviews recommended that pragmatic impairments are universal in autism and comprise a significant element of the disorder. ${ }^{14,15}$ Clinically important diagnostic characteristics of ASD include lack of spontaneous seeking to share enjoyment, emotions, affect, interests, or achievements with other people, and a lack of emotional reciprocity. ${ }^{1-17}$ The present study aimed at the language aspects and revealed similar outcomes found in the previous literatures. ${ }^{15,16}$

In this study we found that $20 \%$ children were able to say name of the known objects and $80 \%$ children could identify $4 / 5$ body parts. Seventy percent children could not make eye contact and understand others facial expression. Ninety percent children could not use "noun" and "pronoun". 90\% parents mentioned 
that their children smiled in response to their activity which he/she likes. Ninety percent children repeatedly utter any words and $70 \%$ parents mentioned that their children use idiosyncratic/neologism words.

ASD is characterized by persistent impairments in social communication and interaction, restricted and repetitive behavior, and an onset of the disorder in earlychildhood. ${ }^{16}$ Outcome of all these studies showed the same observations in the language development which was found in the Bengadeshi children. This study would act as a baseline observation in respect of the language problem in children with autism in Bangladesh.

\section{Conclusion}

Autism spectrum disorder affects the language and communication significantly as the patients with autism have deficiency in language development. This study also tries to find out the extend of these problem in Bangladeshi children with ASD. But with the results of a minimum sample size, city based short duration study a straight cut conclusion would be difficult. Therefore, further large scale multi centered study would be needed to better visualize the problem more efficiently.

\section{References}

1. Sadock BJ, Sadock VA, Ruiz P. Synopsis of psychiatry, $11^{\text {th }}$ edn, 2015. Philadelphia, USA: 1264.

2. Cowen $P$, Harrison $P$, Burns T. Shorter Oxford Textbook of Pshiatry. 6th ed. Oxford University Press; 2012; 663

3. American Psychiatric Association. Diagnostic and Statistical Manual of Mental Disorders, Fifth Edition, Washington DC: American Psychiatric Association, 2013; pp-483.

4. WingL, GouldJ. Severe impairments of social interaction and associated abnormalities in children: epidemiology and classification. J Autism Dev Disord. 1979; 9:11-29.
5. Baird G, Charman T, Baron-CohenS. A screening instrument for autism at 18 months of age: a 6-year follow-up study.] Am Acad Child Adolesc Psychiatry. 2000; 39:694-702.

6. Bertrand J, Mars A, Boyle C, Bove F, Yeargin-Allsopp M, Decoufle P. Prevalence of Autism in a United States Population: The Brick Township, New Jersey, Investigation. Pediatrics. 2001; 108:1155-1161.

7. Chakrabarti S, Fombonne E. Pervasive developmental disorders in preschool children: confirmation of high prevalence.Am J Psychiatry. 2005; 162: 11331141.

8. HondaH, Shimizu Y, Rutter M.No effect of MMR withdrawal on the incidence of autism: a total population study.J Child Psychol Psychiatry. 2005;46: 572-579.

9. Yeargin-Allsopp M, Rice C, Karapurkar T, Doernberg N, Boyle C, Murphy C. Prevalence of Autism in US Metropolitan Area. JAMA. 2003; 289: 49-55.

10. www.sciencedirect.com/science/book/9780126660555

11. AhmedH, Mannan M, Alam F, Rabbani M, Chowdhury N F, Hossain T et. al, Autism in Bangladesh: Current Scenario and Future Prospects.https://www.researchgate.net/.../268143330_Autism_in_Banglades h_Current_Scenari

12. Kim SK. Recent update of autism spectrum disorders. Korean J Pediatr. 2015;58(1):8

13. Lai M-C, Lombardo M V., Auyeung B, Chakrabarti B, Baron-Cohen S. Sex/Gender Differences and Autism: Setting the Scene for Future Research. J Am Acad Child Adolesc Psychiatry. 2015;54(1):11-24.

14. Ben-Yizhak N, Yirmiya N, Seidman I, Alon R, Lord C, Sigman M. Pragmatic Language and School Related Linguistic Abilities in Siblings of Children with Autism. J Autism Dev Disord. 2011;41(6):750-60.

15. Miller M, Young GS, Hutman T, Johnson S, Schwichtenberg AJ, Ozonoff S. Early pragmatic language difficulties in siblings of children with autism: implications for DSM-5 social communication disorder? J Child Psychol Psychiatry. 2015;56(7):774-81.

16. Lartseva A, Dijkstra T, Buitelaar JK. Emotional language processing in autism spectrum disorders: a systematic review. Front Hum Neurosci. 2015;8.

17. Liu K, King M, Bearman PS. Social Influence and the Autism Epidemic. Am J Sociol. 2010;115(5):1387-434. 